\title{
ÍNDICE DE CONFIANZA AL CONSUMIDOR
}

\author{
TATIANA LORENA VAYAS CARRILLO'
}

\section{NACIONAL Y}

\section{POR CIUDADES}

En el presente artículo se realiza un estudio sobre el Índice de Confianza al Consumidor -ICC en el Ecuador y en las 5 provincias donde se levanta la información necesaria para el cálculo de este índice. Los datos necesarios son recopilados por el Instituto Nacional de Estadística y Censos - INEC, mediante la Encuesta Nacional de Empleo, Desempleo y Subempleo - ENEMDU

La muestra mensual es de aproximadamente 3,800 hogares de las ciudades de Quito, Guayaquil, Ambato, Cuenca y Machala. El Índice de Confianza al Consumidor se construye como un promedio ponderado del Índice de Situación Presente y del Índice de Expectativas. Este índice se encuentra acotado entre 0 y 100 puntos. (Banco Central del Ecuador - BCE, Reporte Diciembre, 2015)

Figura 1. Índice de situación presente durante el 2015

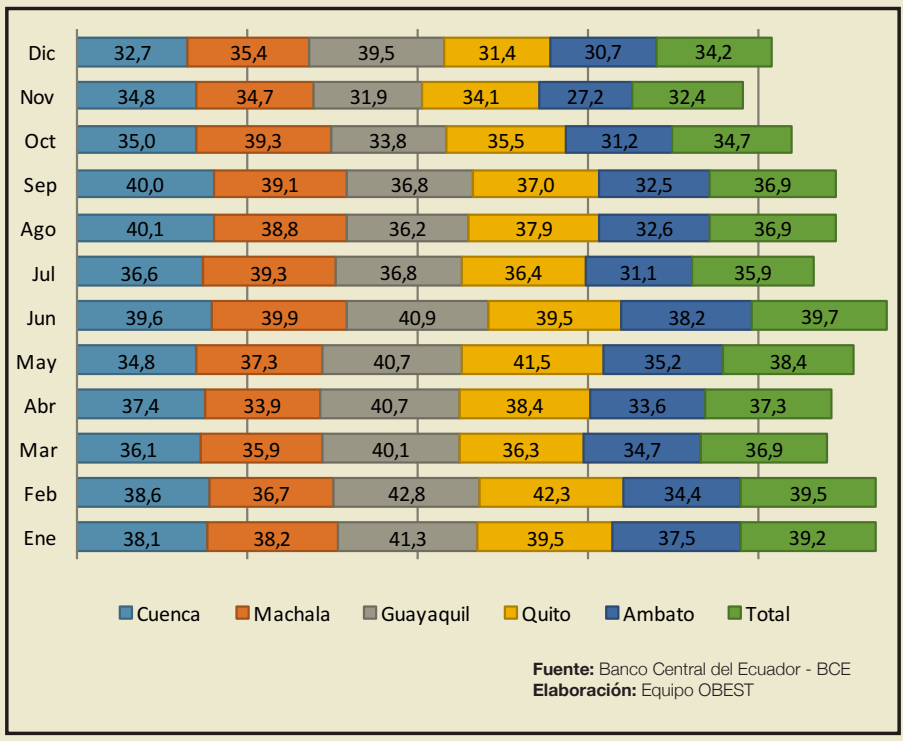

El Índice de Situación Presente se construye en base a tres preguntas: 1) ¿En relación al mes anterior la situación económica de su hogar es? con las opciones mejor, igual, peor, 2) ¿En relación al mes anterior el consumo de su hogar en entretenimiento es? Las alternativas mayor, igual, menor, 3) ¿En relación al mes anterior la situación económica del país es? opciones mejor, igual, peor (BCE, Reporte Diciembre, 2015).

A nivel nacional durante enero - diciembre 2015 la ciudadanía opina que la situación actual del país se encuentra en promedio en 36,8 puntos/100, destacando noviembre, mes en el que la población incrementó la opinión pesimista a 32,4 puntos; caso contrario en junio que disminuyó la opinión pesimista a 39,7 puntos.

Cabe destacar que noviembre en Ambato registra el nivel más bajo del índice con 27,2 puntos entre las 5 ciudades donde se realiza el levantamiento de la información, en cambio en Guayaquil en el mes de febrero se registra el mes más alto con 42.8 puntos.

${ }^{1}$ Economista - Analista de Investigación y Desarrollo del Observatorio Económico y Social de Tungurahua - UTA
Figura 2. Índice de Expectativas Futuras

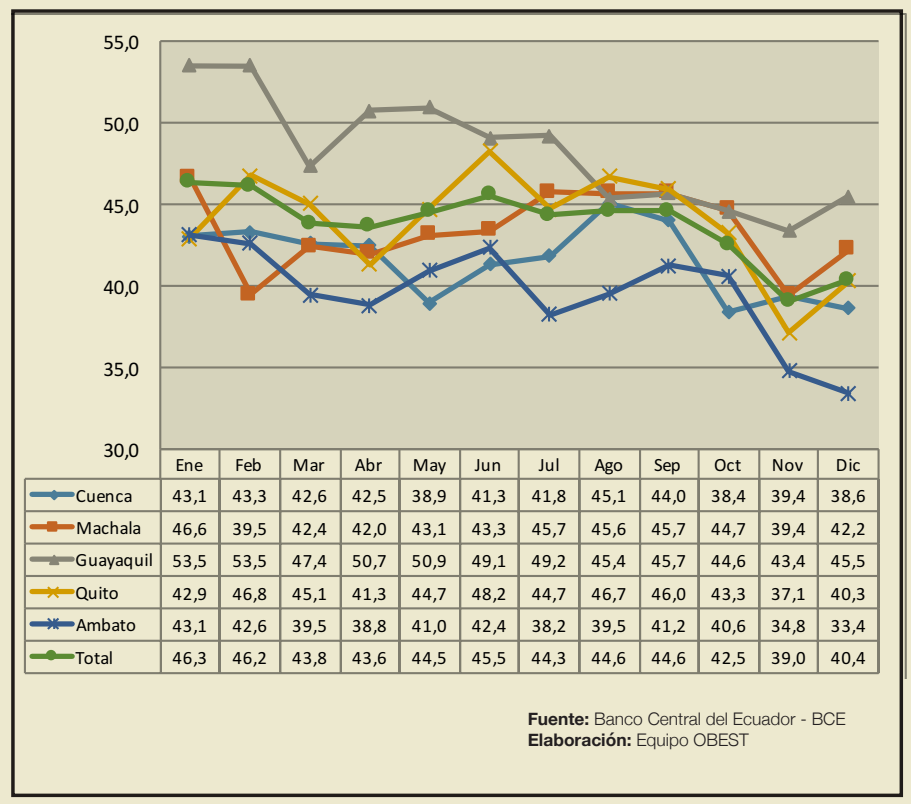

El Índice de Expectativas Futuras se construye en base a tres preguntas: 1) ¿Cómo cree usted que será la situación económica de su hogar en los próximos tres meses?, 2) ¿Cómo cree usted que será el consumo de su hogar en entretenimiento en los próximos tres meses?, 3) ¿Cómo considera que será la situación económica del país durante los próximos 3 meses? (BCE, Reporte Diciembre, 2015)

El Índice de Expectativas Futuras en total de las cinco ciudades muestra un promedio de 43,8 puntos, en la figura 2, se puede observar que los hogares encuestados en Guayaquil son menos pesimistas ya que en enero obtiene 53 puntos (puntaje más alto) y cierra en diciembre con 45,5 puntos, caso contrario se identifica en Ambato donde los ciudadanos fueron en cierto punto más pesimistas en enero a pesar de ser el más alto 43,1 puntos durante el año y el más bajo de 33,4 puntos, siendo este el menor en las cinco ciudades encuestadas. Es importante señalar que a inicios del año las expectativas futuras de la ciudadanía eran mejores pero con el pasar los meses ha ido decreciendo.

UNIVERSIDAD

TÉCNICA DE AMBATO 
Figura 3. Índice de Confianza del Consumidor

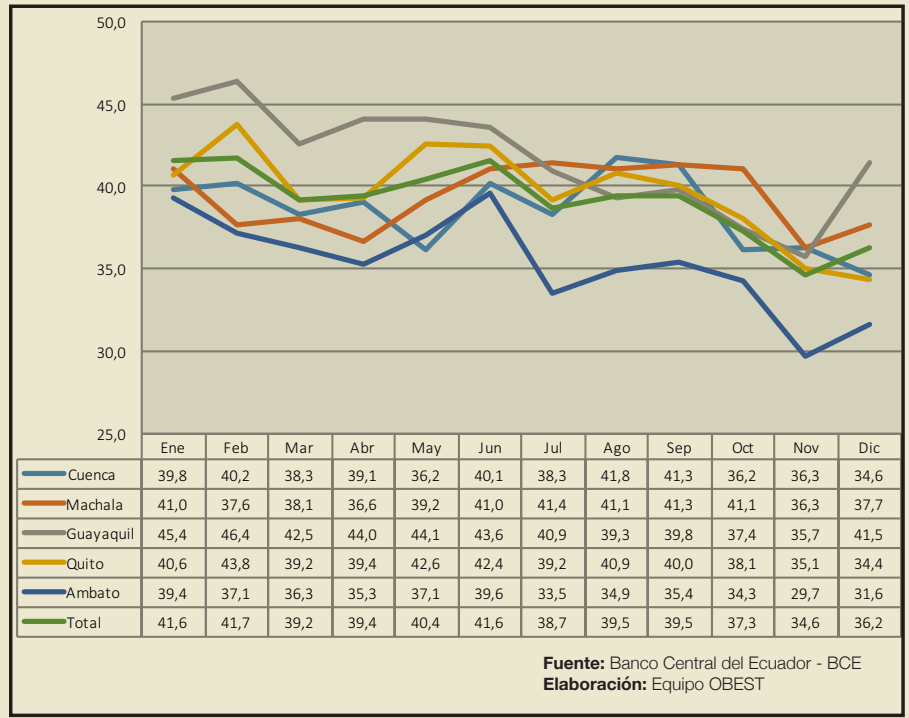

El ICC define el nivel de optimismo de los hogares respecto de sus percepciones actuales y expectativas futuras en relación al consumo, a la situación económica del hogar y al escenario económico del país, convirtiéndose en un indicador clave del estado general de la economía; el ICC se encuentra acotado entre 0 y 100 puntos, siendo 0 un reflejo de una percepción completamente negativa ante el porvenir económico del hogar y del país y 100 un reflejo de perfecto optimismo al escenario económico del hogar y del país. (EUMED, Galo Viteri López)

Al igual que el Índice de Situación Presente y de Expectativas, en el Índice de Confianza al Consumidor las ciudades que se destacan son Guayaquil y Ambato; la primera con una opinión menos pesimista que se refleja en la figura 3, enero inicia con 45,4 puntos pero conforme pasan los meses disminuye, al terminar el año tienen un leve incremento en diciembre cerrando con 41,5 puntos; la segunda ciudad, es decir, Ambato con una opinión más pesimista ya que en la mayoría de los meses refleja un puntaje por debajo de las demás ciudades, siendo noviembre el mes que menor puntaje presenta con 29,7puntos; caso contrario sucede con el mes de junio con 39,6 puntos.

Figura 4. Comparativo de Ambato de índices del mes de enero de 2013-2016

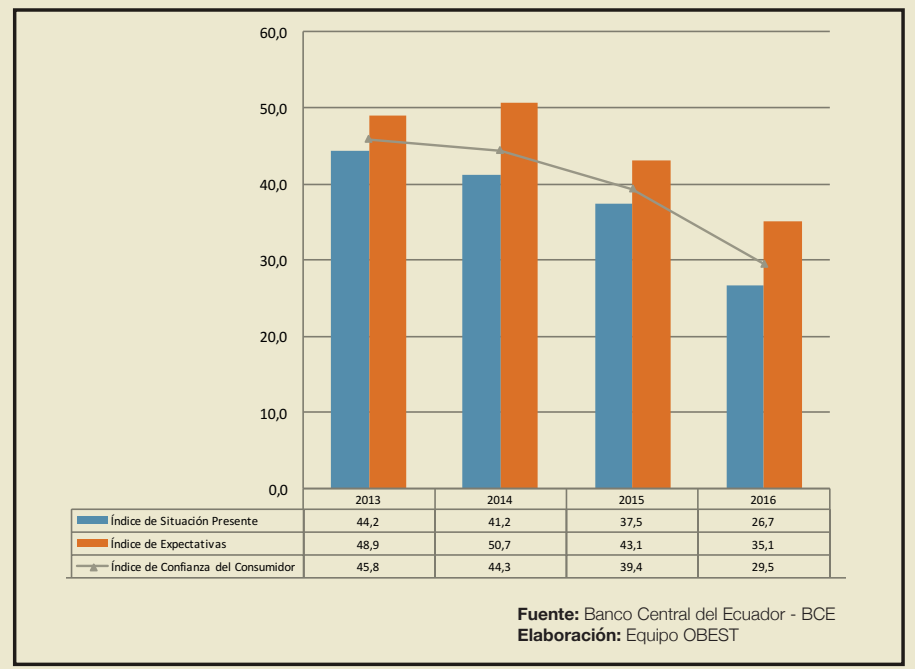

En la figura 4, se puede determinar que en el periodo 2013 -2016, los índices han decrecido en la ciudad de Ambato, es decir, que el optimismo de los consumidores ambateños cada año disminuye, la situación actual con respecto al 2013 muestra una diminución de 17,5 puntos, es decir, $40 \%$ menos, las expectativas del futuro bajan en 13,8 puntos, esto es $29 \%$ y finalmente el índice de confianza al consumidor decrece en 16,3 puntos lo que representa un $36 \%$.

\section{Figura 5. Indicadores según nivel de instrucción a nivel Nacional, enero 2016}

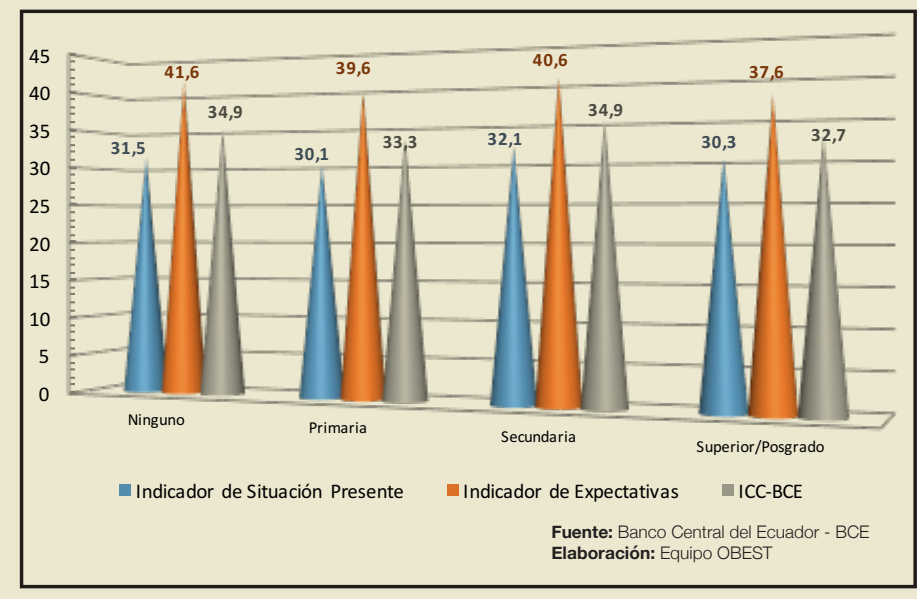

La figura 5, muestra que las personas que no tienen ningún nivel de estudios, tienen una opinión menos pesimista en el futuro 41,6 puntos, a diferencia de las personas con estudios superior o posgrado que ven el futuro con otras perspectivas, 37,6 puntos, algo similar sucede con el índice de situación presente y el índice de confianza al consumidor ICC.

\section{Figura 6. Indicadores por situación laboral a nivel Nacional Enero 2016}

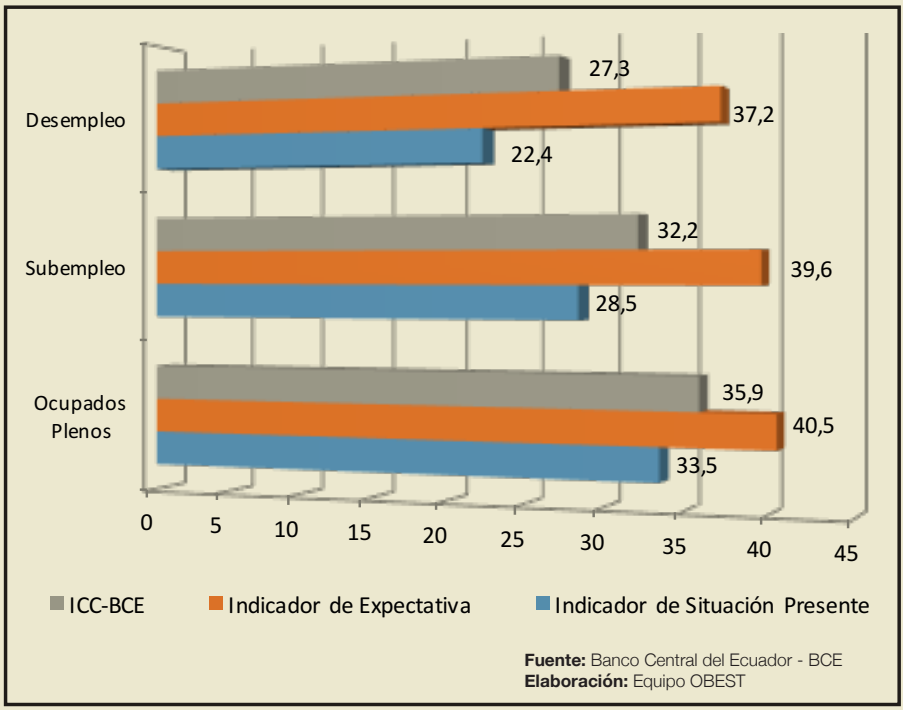

Es importante recalcar que mientras la puntuación se acerca a cero (0) la percepción es pesimista y si se acerca a cien (100) el puntaje es de optimismo. Los desempleados perciben la situación presente con 22,4 puntos, se debe que al momento de la encuesta no tienen empleo por lo que la situación que ellos viven es difícil, de igual manera las perspectivas del futuro sin empleo no son buenas por lo que presenta 37,2 puntos, se puede evidenciar una diferencia entre el desempleo y los ocupados plenos. 
Los ocupados plenos tienen una perspectiva del Índice de Situación Presente de 11,4 puntos más que los desempleados, y las perspectivas del futuro son 3,3 puntos adicionales que las personas sin empleo y finalmente el Índice de Confianza al Consumidor con 7,7 puntos adicionales.

Figura 7. Indicadores por categoría de ocupación a nivel Nacional, enero 2016

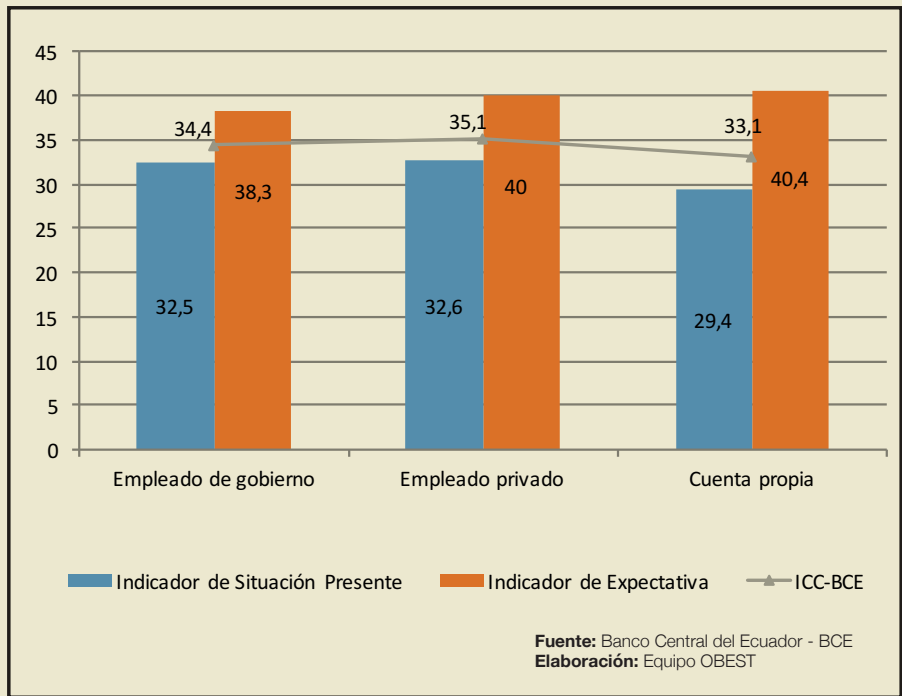

En la figura 7, se determina que los ciudadanos que trabajan por cuenta propia incrementan su opinión pesimista en los tres índices y los que consideran una mejor situación son los empleados privados, caso contrario sucede con los empleados públicos

Figura 8. Indicadores por sectores a nivel Nacional, enero 2016

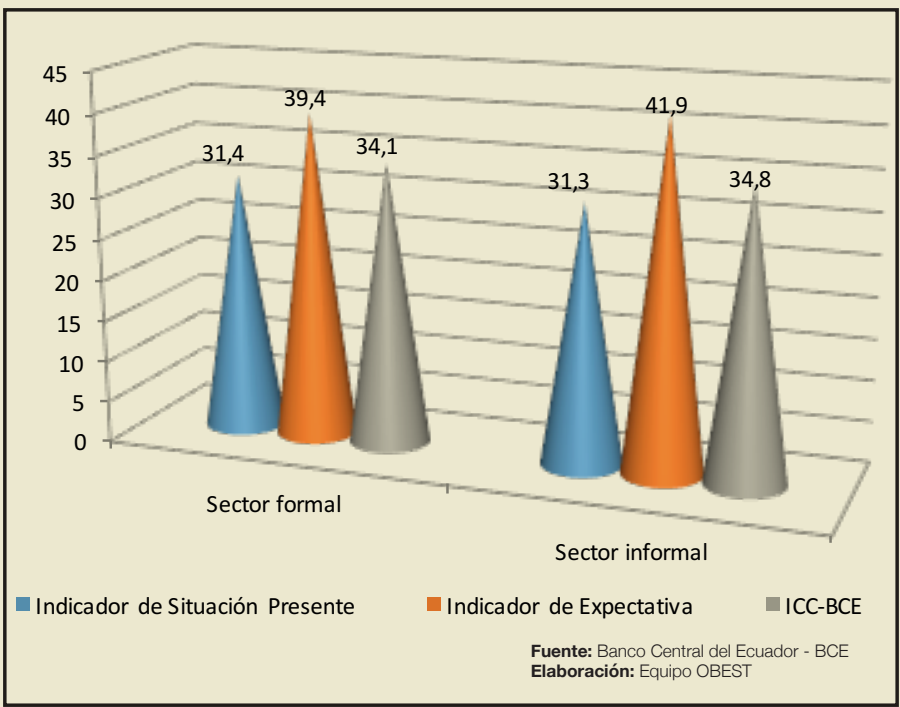

La figura 8, refleja que el Índice de Situación Presente es mayor en el sector formal con 31,4 puntos; caso contrario sucede con el Índice de Expectativas y el de Confianza al consumidor con 41,9 y 34,8 respectivamente del sector informal.

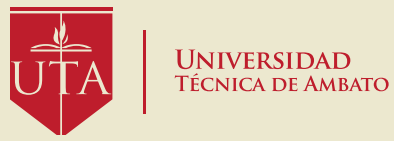

Figura 9. Personas que piensan adquirir un inmueble en los Próximos 6 meses

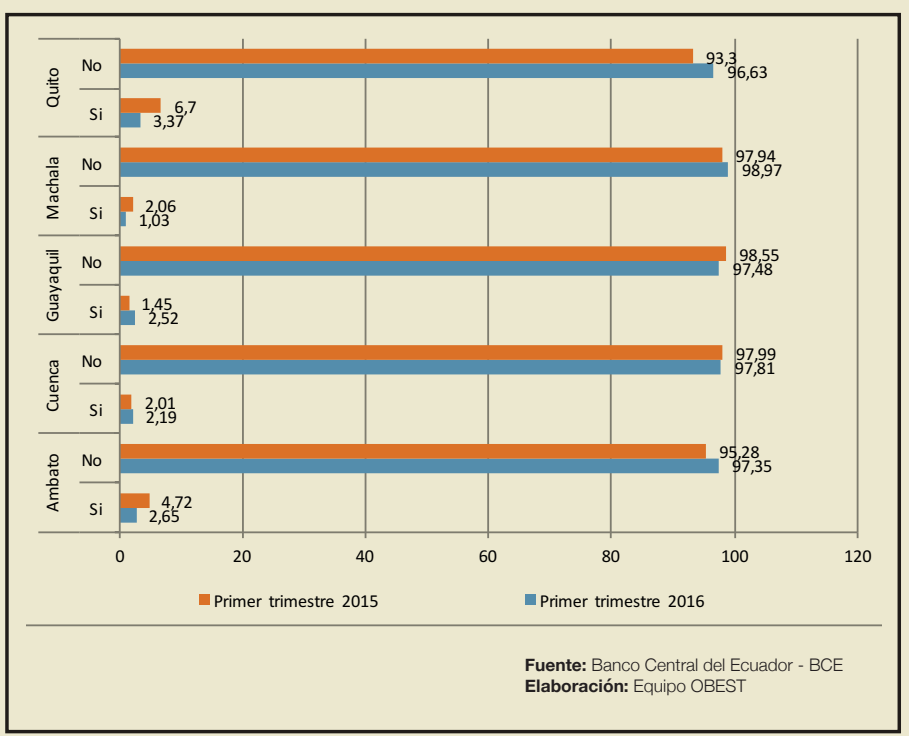

En la figura 9, se encuentra un comparativo del primer trimestre del 2015 y 2016, se destaca que ha disminuido el porcentaje de personas que desean comprar un inmueble en las ciudades de Ambato, Machala y Quito aproximadamente a la mitad y ha incrementado en Cuenca y Guayaquil.

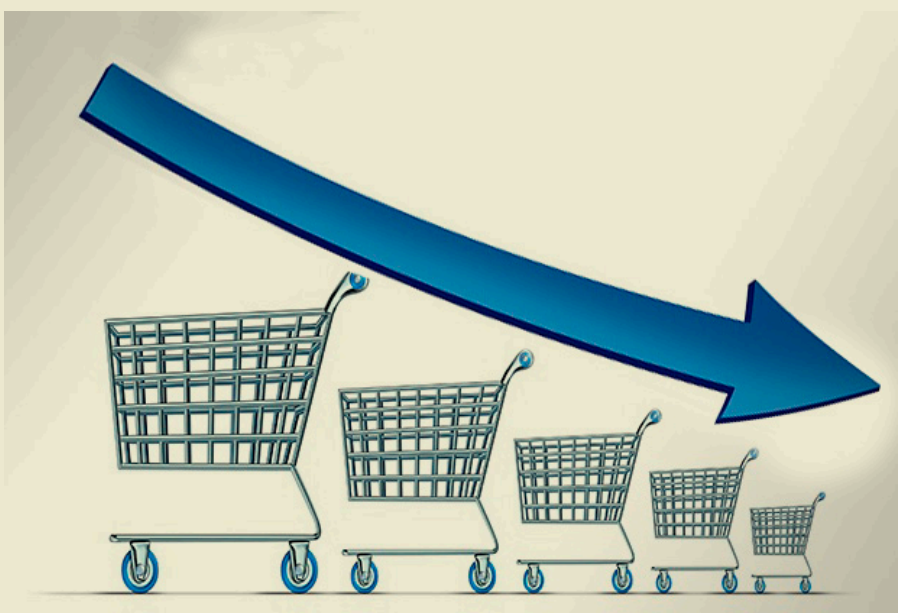

Referencias:

Referencias

- Banco Central del Ecuador - BCE. Recuperado en: http://contenido.bce.fin.ec/documentos/Estadisticas/SectorReal/Previsiones/IndCoyuntura/mensual/ICC_indice.htm

- Banco Central del Ecuador - BCE., reporte Diciembre, 2015. Recuperado en: http://contenido. bce.fin.ec/documentos/Estadisticas/SectorReal/Previsiones/IndCoyuntura/mensual//CC201512.pdf - VITERI, G., La Economía Ecuatoriana en el periodo 2006 - 2011. Recuperado en: http://www. eumed.net/libros-gratis/2012b/1218/indice_confianza.htm 